\title{
COULOMB EXCITATION BY 45 MeV ALPHA PARTICLES*
}

\author{
F. T. BAKER and R. TICKLE \\ Cyclotron Laboratory, Department of Physics, \\ The University of Michigan, Ann Arbor, Michigan 48105, USA
}

Received 8 April 1970

\begin{abstract}
Cross sections for inelastic scattering of $45 \mathrm{MeV} \alpha$ particles from ${ }^{140} \mathrm{Ce}$ and ${ }^{208} \mathrm{~Pb}$ have been meas ured for angles as small as $8^{\circ}$. Coulomb excitation has been found to be a significant contributor to the cross sections for excitation of $2^{+}$and $3^{-}$levels.
\end{abstract}

The effect of Coulomb excitation on inelastic scattering is most pronounced at small angles. Because of the large number of partial waves necessary to perform the DWBA analysis of smallangle data, this effect has not been carefully examined. Recent expansion of the distorted wave code DWUCK [1] to include 102 partial waves has made it possible to analyse at small angles the inelastic scattering of $45 \mathrm{MeV} \alpha$ particles from $140 \mathrm{Ce}$ and $208 \mathrm{~Pb}$.

The $45 \mathrm{MeV} \alpha$-particle beam from the University of Michigan cyclotron was transported to the scattering chamber through two beam preparation magnets which have an intermediate focus; this arrangement provided a well-focused, well-resolved beam on the target without the use of slits in the scattering chamber, thus greatly reducing the background at small angles. The scattered particles were magnetically analysed and detected either in nuclear emulsions or with a position sensitive detector. The $208 \mathrm{~Pb}$ target (enriched to $99.3 \%$ ) was $800 \mu \mathrm{g} / \mathrm{cm}^{2}$ thick and the thicknesses of the natural cerium targets $(88.5 \% 140 \mathrm{Ce})$ ranged from 300 to $1000 \mu \mathrm{g} / \mathrm{cm}^{2}$. The $1.66 \mathrm{MeV}$ $3^{-}$level in the impurity $142 \mathrm{Ce}$ was not clearly resolved from the stronger group corresponding to the $1.597 \mathrm{MeV} 2^{+}$level in ${ }^{140} \mathrm{Ce}$. From a highresolution spectrum it was determined that the intensity of the $3^{-}$level in ${ }^{142} \mathrm{Ce}$ was $14 \%$ of the intensity of the $2.464 \mathrm{MeV}^{-}$level in $140 \mathrm{Ce}$. If one assumes that the two $3^{-}$angular distributions are identical, the intensity of the $2^{+}$level in $140 \mathrm{Ce}$ can be separated from that of the closelying $3^{-}$I evel in $142 \mathrm{Ce}$.

The DWBA calculations for the inelastic scat-

* Work supported in part by the U.S. Atomic Energy Commission. tering assumed a collective model for the nuclear part of the interaction [2]. A six-parameter optical potential was used for ${ }^{140} \mathrm{Ce}$ and the form factor for inelastic scattering was calculated with equal deformation lengths $\beta_{J} R$ for real and imaginary wells. Coulomb excitation was included as in ref. [2].

The adequacy of 102 partial waves was tested by examining the convergence of the predicted cross section as the number of partial waves was varied. Fig. 1 shows how the cross section predicted for excitation of the $2^{+}$state in $140 \mathrm{Ce}$ depends on the number of partial waves. Although

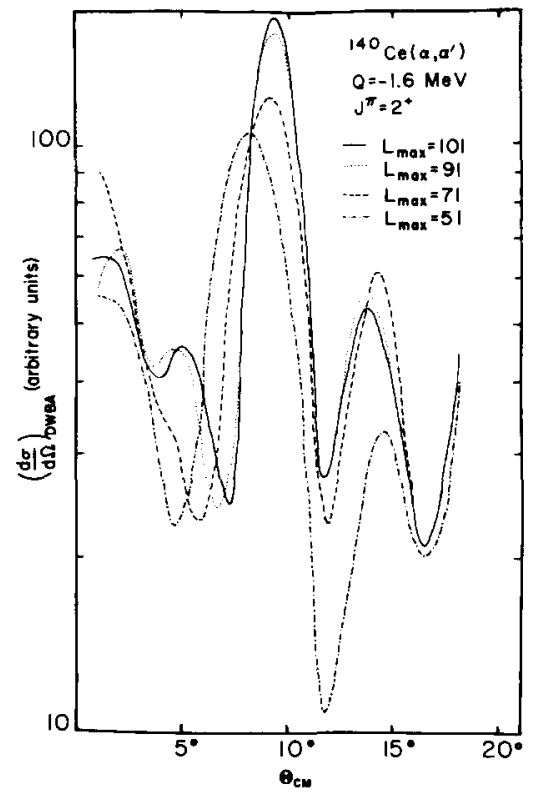

Fig. 1. DWBA calculations, including Coulomb excitation, for a transition to the first $2^{+}$level in ${ }^{140} \mathrm{Ce}$. 
the calculation has not yet fully converged for 102 partial waves, the difference between 92 and 102 partial waves is small and little change in the predicted cross section is expected for increased numbers of partial waves. The calculations for the $3^{-}$levels in ${ }^{140} \mathrm{Ce}$ and ${ }^{208} \mathrm{~Pb}$ were found to converge for all angles where cross section measurements were made and the predictions are expected to be accurate.

The results of this experiment for the strong $2^{+}$and $3^{-}$excitations of $140 \mathrm{Ce}$ are shown in fig. 2. The main effect of Coulomb excitation on the $3^{-}$level is to deepen the minimum at $18^{\circ}$. This prediction is seen to conform quite well to the experimental data. The interference between

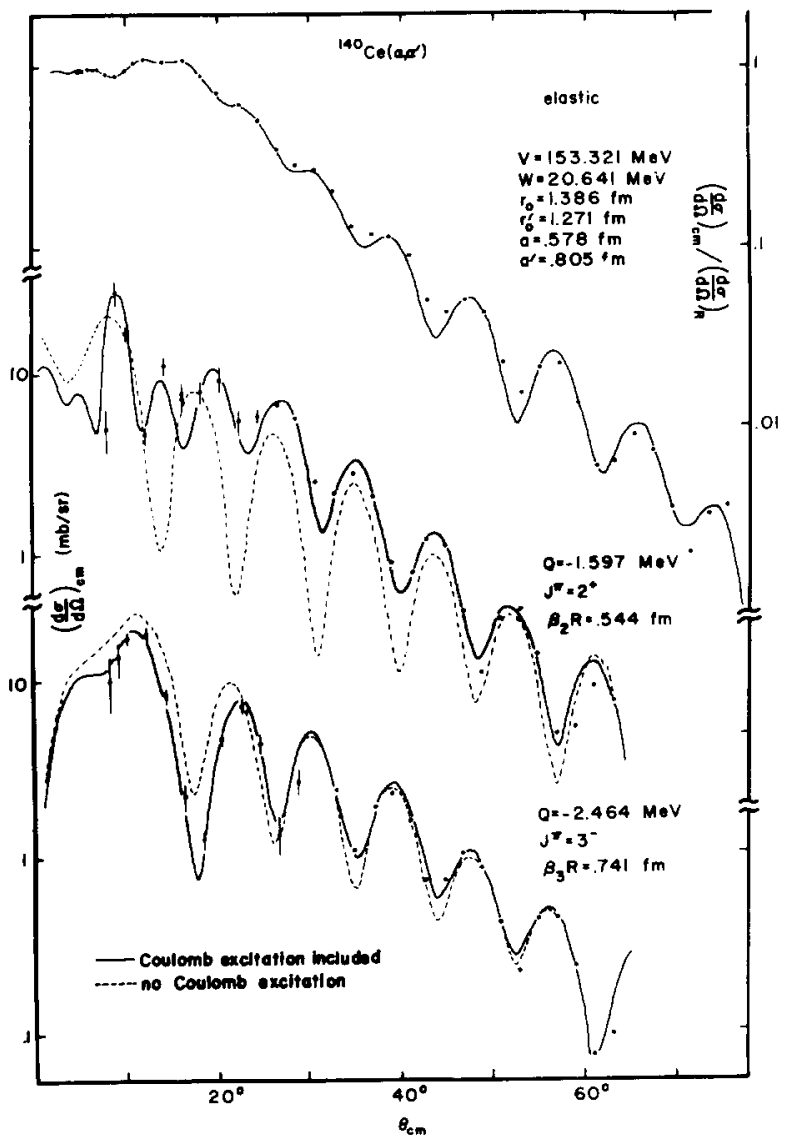

Fig. 2. Angular distributions for elastic and inelastic scattering from $140 \mathrm{Ce}$. Indicated errors are due to both statistics and background subtraction. The same values of $\beta_{J} R$ were used for calculations with and without Coulomb excitation. The optical potential used to fit the elastic scattering was

$$
\begin{aligned}
& U(r)=-V\left(\mathrm{e}^{x+1}\right)^{-1}-\mathrm{i} W\left(\mathrm{e}^{x^{\prime}+1}\right)^{-1}, \\
& x=\left(r-r_{\mathrm{O}} A^{1 / 3}\right) / a, x^{1}=\left(r-r_{\mathrm{O}}^{\prime} A^{1 / 3}\right) / a .
\end{aligned}
$$

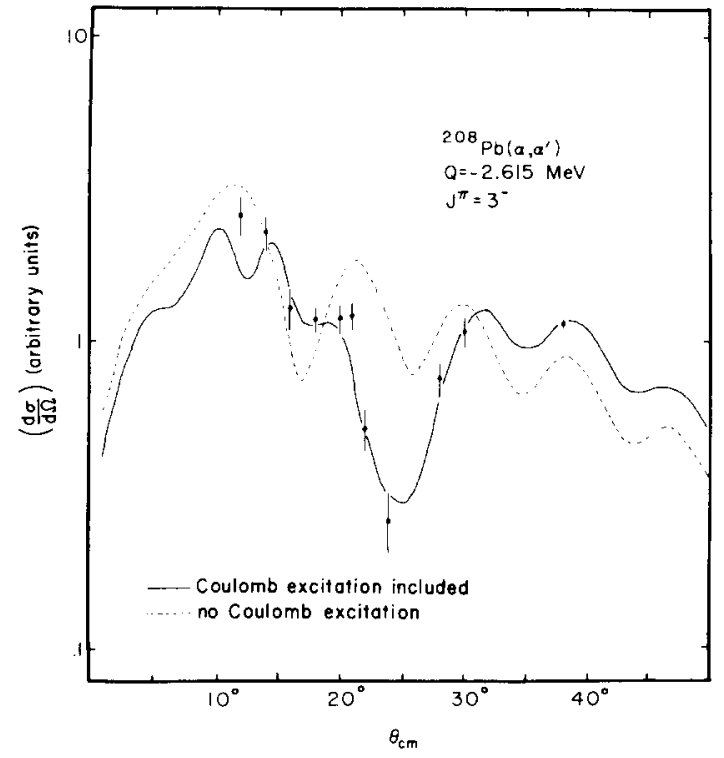

Fig. 3. The angular distribution for the excitation of the $2.615 \mathrm{MeV}$ level in $208 \mathrm{~Pb}$. Indicated errors are due to both statistics and background subtraction. The same normalization was used for calculations with and without Coulomb excitation.

nuclear and Coulomb interactions is much more pronounced for excitation of the $2^{+}$level. The shape of the predicted cross section below $30^{\circ}$ is greatly altered by the inclusion of Coulomb excitation and the DWBA prediction is in quite good agreement with the experimental data. Also of interest is the difference between the magnitudes of the predicted cross sections at larger angles where the shapes are qualitatively similar; hence failure to account for Coulomb excitation will alter the deduced values of deformation lengths. Since the form factor for Coulomb excitation falls off as $r^{-J-1}$, the effects of Coulomb excitation on $4^{+}$and $5^{-}$states in the spectrum were too small to be observed experimentally.

The results for excitation of the $2.615 \mathrm{MeV}^{-}$ level in ${ }^{208} \mathrm{~Pb}$ are shown in fig. 3 . The optical parameters for $208 \mathrm{~Pb}$ were those of the $V=114.3$ $\mathrm{MeV}$ family of ref. [3]. Measurements at $42 \mathrm{MeV}$ and $43 \mathrm{MeV}$ which verify the presence of the deep minimum at $25^{\circ}$ have previously been performed $[3,4]$. The present experiment extends the data to $12^{\circ}$ and shows that Coulomb excitation alters the cross section for the $3^{-}$state in $208 \mathrm{~Pb}$ more strongly than for the $3^{-}$state in $140 \mathrm{Ce}$. Again note that omission of Coulomb excitation alters the deduced value of the deformation length.

In conclusion, we have shown that the effects of Coulomb excitation are important even when the $\alpha$-particle energy is well above the Coulomb 
barrier. Coulomb excitation not only alters the shape of the cross section at small angles, but may also change the magnitude of the predicted cross section at larger angles. In the cases considered, interference between nuclear and Coulomb processes seems well described by the DWBA formalism.
References

[1] P. D. Kunz, University of Colorado, unpublished.

[2] R. H. Bassel, G. R. Satchler, R. M. Drisko and E. Rost, Phys. Rev. 128 (1962) 2693.

[3] G. R. Satchler, H. W. Broek and J. L. Yntema, Phys. Letters 16 (1965) 52.

[4] J. Alster, D. L. Hendrie and R. J. Peterson, Cyclotron Progress Report. University of Washington (1964). 